\title{
recillunds
}

Revista Cientifica Mundo de la Investigación y el Conocimiento

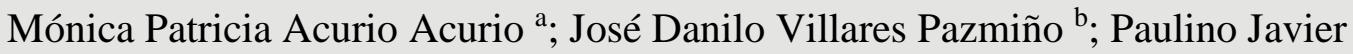 \\ Suarez Guamán ${ }^{\mathrm{c}}$
}

Uso de las redes sociales para actividades académicas colaborativas en la educación superior

Use of social networks for collaborative academic activities in higher education

Revista Científica Mundo de la Investigación y el Conocimiento. Vol. 3 núm.3, septiembre, ISSN: 2588-073X, 2019, pp. 3-30

DOI: $10.26820 /$ recimundo/3.(3).septiembre.2019.3-30

URL: http://recimundo.com/index.php/es/article/view/508

Código UNESCO: 3205 Medicina Interna

Tipo de Investigación: Artículo de Revisión

Editorial Saberes del Conocimiento

Recibido: 15/05/2019

Aceptado: 23/06/2019

Publicado: 30/09/2019

Correspondencia: jvillares@utb.edu.ec

a. Magister en Gerencia Educativa; Diploma Superior en Docencia Universitaria; Ingeniera en Sistemas; Facultad de Ciencias de la Salud; Carrera de Optometría; Universidad Técnica de Babahoyo; macurio@utb.edu.ec

b. Magister en Gestión de Bases de Datos; Ingeniero en Sistemas; Facultad de Administración, Finanzas e Informática; Carrera de Ingeniería en Sistemas; Universidad Técnica de Babahoyo; jvillares@utb.edu.ec

c. Magister en Informática Empresarial; Especialista en Redes de Comunicación de Datos; Diploma Superior en Sistemas de Información Empresarial; Ingeniero en Sistemas; Facultad de Ciencias de la Salud; Carrera de Enfermería; Universidad Técnica de Babahoyo; jsuarez@utb.edu.ec 


\section{Uso de las redes sociales para actividades académicas colaborativas en la educación superior}

Vol. 3, núm. 3., (2019)

Mónica Patricia Acurio Acurio; José Danilo Villares Pazmiño; Paulino Javier Suarez Guamán

\section{RESUMEN}

El proceso evolutivo del conocimiento ha venido de la mano del desarrollo de investigaciones y estas a su vez han servido de herramientas para el desarrollo de los diferentes enfoques pedagógicos de la educación. La utilización de herramientas pedagógicas para transferir el conocimiento ha sido de vital importancia ya que allí se genera el conocimiento sobre temas en específico. Estas herramientas han evolucionado a través del tiempo y han permitido acercar más a los individuos al conocimiento que desean adquirir quebrando viejos paradigmas incrustados en la sociedad. Es aquí donde entran las TIC como herramientas de desarrollo del conocimiento. Las redes sociales están jugando un papel importante en la masificación de conocimiento en este mundo globalizado. Por este motivo, esta investigación tiene como objetivo general analizar el uso de las redes sociales para actividades académicas colaborativas en la educación superior. La metodología empleada se basó en la utilización de investigación documental y bibliográfica. Los resultados que se obtuvieron se basaron en las características de las TIC, la realidad de la universidad ecuatoriana con las TIC y las redes sociales y las universidades ecuatorianas. Como conclusión se obtuvo que el uso de las redes sociales permite la masificación del conocimiento, a través de las distintas plataformas virtuales, haciendo que los nuevos profesionales tengan mayor pertenencia en su labor y aunado a esto un mayor compromiso social ya que fomentan el autoaprendizaje, desarrollan habilidades, fomentan el pensamiento crítico y comprenden la existencia de la diversidad.

Palabras Claves: Investigación; conocimiento; TIC; redes sociales. 


\title{
Uso de las redes sociales para actividades académicas colaborativas en la educación superior
} Vol. 3, núm. 3., (2019)

Mónica Patricia Acurio Acurio; José Danilo Villares Pazmiño; Paulino Javier Suarez Guamán

\begin{abstract}
The evolutionary process of knowledge has come hand in hand with the development of research and these in turn have served as tools for the development of different pedagogical approaches to education. The use of pedagogical tools to transfer knowledge has been of vital importance since there is generated knowledge on specific topics. These tools have evolved over time and have allowed individuals to get closer to the knowledge they wish to acquire by breaking old paradigms embedded in society. This is where ICTs come in as tools for knowledge development. Social networks are playing an important role in the massification of knowledge in this globalized world. For this reason, this research has as its general objective to analyze the use of social networks for collaborative academic activities in higher education. The methodology used was based on the use of documentary and bibliographic research. The results obtained were based on the characteristics of ICT, the reality of the Ecuadorian university with ICT and social networks and Ecuadorian universities. As a conclusion it was obtained that the use of social networks allows the massification of knowledge, through the different virtual platforms, making new professionals have greater belonging in their work and coupled with this a greater social commitment as they promote self-learning, they develop skills, foster critical thinking and understand the existence of diversity.
\end{abstract}

Key Words: Research; knowledge; ICT; social networks. 


\section{Uso de las redes sociales para actividades académicas colaborativas en la educación superior}

Vol. 3, núm. 3., (2019)

Mónica Patricia Acurio Acurio; José Danilo Villares Pazmiño; Paulino Javier Suarez Guamán

\section{Introducción.}

Uno de los principales procesos para obtener una información es poder realizar una investigación que conlleve a la solución de un problema o situación. Esto se ha aplicado en todas las etapas evolutivas del ser humano. Desde la necesidad de poder alimentarse hasta poder construir un refugio. La investigación ha sido parte fundamental del desarrollo de la humanidad. Esto conlleva a que la investigación sea parte del proceso educativo del individuo. Investigar es un proceso inherente a la vida misma; no obstante, la investigación en educación conlleva, además de un interés y una necesidad, una búsqueda para la profundización y comprensión de los fenómenos educativos, más allá de lo meramente evidente (Pereira Pérez, 2011).

Por lo cual es necesario poder determinar cuál es la importancia de la investigación del contexto general.

“La investigación se puede definir también como la acción y el efecto de realizar actividades intelectuales y experimentales de modo sistemático con el propósito de aumentar los conocimientos sobre una determinada materia y teniendo como fin ampliar el conocimiento científico, sin perseguir, en principio, ninguna aplicación práctica”. (Ortiz Flores \& Bernal Zepeda, 2007, pág. 2)

Es decir, se deben aplicar procedimientos, dentro del marco investigativo, con el fin de poder obtener los conocimientos necesarios para la solución de un problema en específico. Es necesario llevar a cabo un desarrollo metódico que permita la adecuada consecución de los objetivos propuestos, así como una formulación clara, concreta y precisa del problema y una metodología de investigación rigurosa y adecuada al tipo de trabajo (López Noguero, 2002). 


\section{Uso de las redes sociales para actividades académicas colaborativas en la}

educación superior Vol. 3, núm. 3., (2019)

Mónica Patricia Acurio Acurio; José Danilo Villares Pazmiño; Paulino Javier Suarez Guamán

Este desarrollo metodológico va enmarcado en la aplicación de un método científico debido a las etapas o fases por las cuales puede pasar el desarrollo investigativo. Aprender y aplicar el método científico es esencial para la formación y el desempeño de un profesional, ya que en su trabajo diario tiene que recoger información, procesar los datos y plantearse hipótesis y establecer diagnósticos (Carrizo Estévez, 2010).

Poder aplicar el desarrollo metodológico de un proceso de investigación se tiene que vencer paradigmas dentro de la enseñanza de la educación. Cuando el investigador se encuentra en la etapa inicial de la formulación de un proyecto de investigación es indispensable conocer y posicionarse en un determinado paradigma que guíe el proceso investigativo como lo son: positivismo, pospositivismo, teoría crítica y constructivismo (Ramos, 2015). Esto se puede manifestar en que los paradigmas que orientan la práctica docente habitual son concretamente. la enseñanza "por transmisión de conocimientos ya elaborados\# y la enseñanza por “descubrimiento inductivo y autónomo" (GIL PEREZ, 1983). Lo que conlleva a que se determinen los enfoques de la enseñanza manifestados en la Figura 1. 


\section{Uso de las redes sociales para actividades académicas colaborativas en la educación superior}

Vol. 3, núm. 3., (2019)

Mónica Patricia Acurio Acurio; José Danilo Villares Pazmiño; Paulino Javier Suarez Guamán

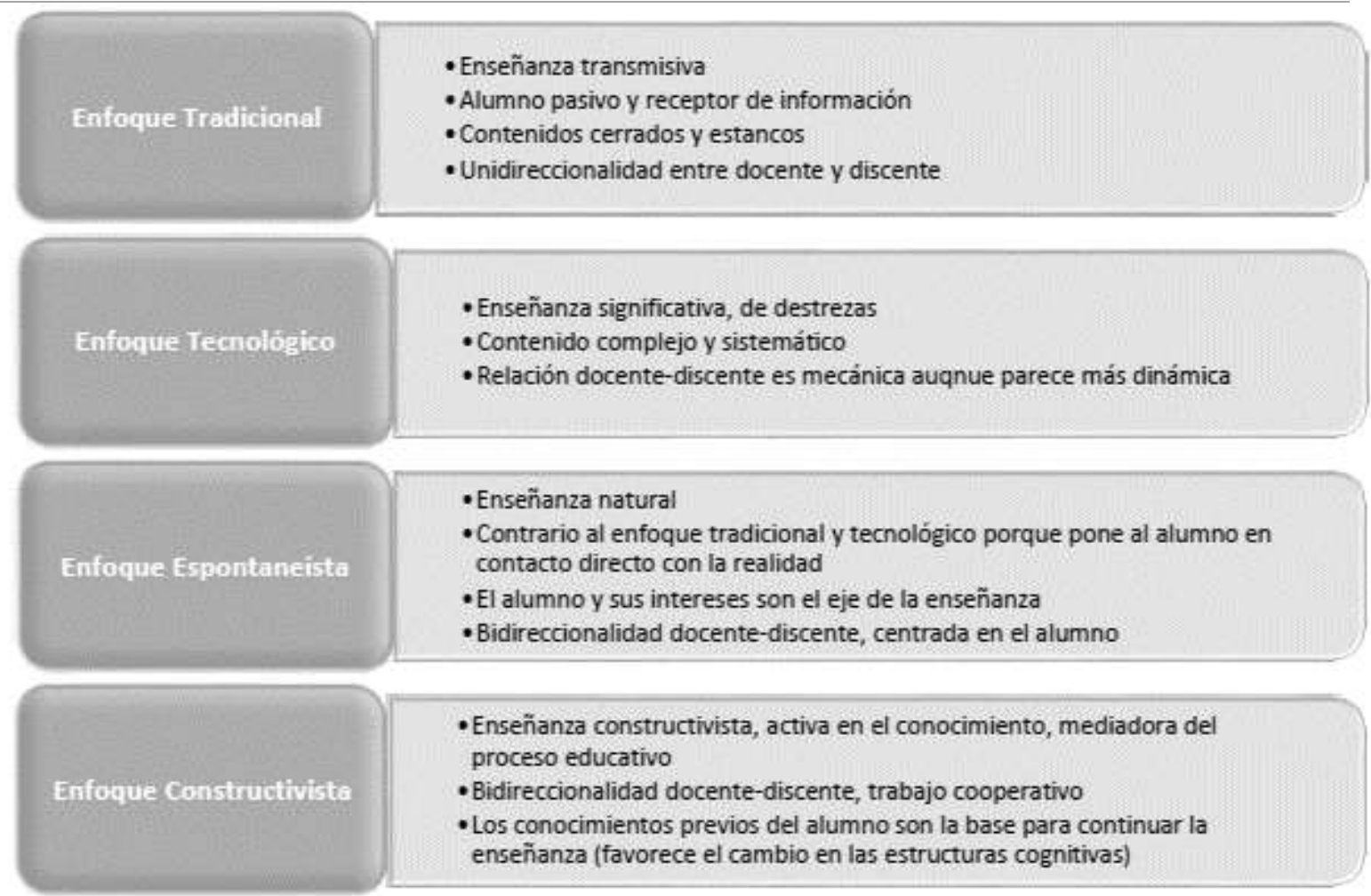

Figura 1. Enfoques de enseñanza

Fuente: (Renés Arellano, 2018)

Dependiendo del tipo de enfoque de enseñanza que ofrezca el docente será la capacidad de desarrollo investigativo realizado por el estudiante. Es aquí donde deben plantearse enfoques que no solo busque el desarrollo profesional sino también personal y social. Estos cambios de perspectiva en relación con la cultura docente deben posibilitar el planteo de la formación universitaria no sólo en clave profesional sino también ciudadana (Martínez Martín, 2006).

Estos cambios culturales deben ir enmarcados en la búsqueda de nuevas herramientas o métodos en las cuales la enseñanza sea más directa y que permita que la investigación sea más amplia. Es por ello, que las universidades deben ser rápidas y capaces. Esto se logra mediante 


\section{Uso de las redes sociales para actividades académicas colaborativas en la educación superior}

Vol. 3, núm. 3., (2019)

Mónica Patricia Acurio Acurio; José Danilo Villares Pazmiño; Paulino Javier Suarez Guamán una organización premeditada, de explotar más eficazmente lo que ya saben hacer y explorar nuevos proyectos que podrían serles útiles, en pocas palabras, más capaces de encontrar nuevas formas de proceder que puedan combinarse con procedimientos tradicionales (Clark, 1998).

Poder lograr que estos dos parámetros se puedan integrar a la universidad tradicional a sido bastante complejo debido a que se deben vencer paradigmas dentro del claustro universitario, desde el docente hasta las autoridades que la rigen. Es aquí donde entran las tecnologías de la información y comunicación (TIC). Estas se usan de forma habitual dentro de nuestras configuraciones sociales, ya que se encuentran presentes en nuestro mundo, forman parte de nuestra cultura, de nuestra vida (BAELO ÁLVAREZ \& CANTÓN MAYO, 2009).

La tecnología aplicada a la investigación y por ende a la información es un complemento de esta en la actualidad.

"La información es el contenido; lo digital, su continente. La información aparece en todas partes, entre otras razones, porque la tecnología está omnipresente en nuestra sociedad. La tecnología digital cobra sentido, significación y utilidad social porque nos proporciona experiencias valiosas con la información. Y esta, a su vez, necesita de la tecnología para poder almacenarse, organizarse, replicarse, difundirse, transformarse y ser accesible". (Area Moreira, 2010, pág. 1)

Es por ello, que el internet juega un pale importante para el desarrollo de la investigación e información en la actualidad ya que puede dar soluciones variadas en la consecución del conocimiento. Por lo cual, la aplicación del aprendizaje virtual esta sometido a tres criterios fundamentales: 


\section{Uso de las redes sociales para actividades académicas colaborativas en la educación superior}

Vol. 3, núm. 3., (2019)

Mónica Patricia Acurio Acurio; José Danilo Villares Pazmiño; Paulino Javier Suarez Guamán

“a) que se realice en red, lo que permite una actualización inmediata, almacenaje y recuperación, distribución y poder compartir los contenidos y la información; b) que se haga llegar al usuario final a través de un ordenador utilizando estándares tecnológicos de Internet; y c) que esté centrado en la más amplia visión de soluciones al aprendizaje que vayan más allá de los paradigmas tradicionales de la formación”. (Rosenberg, 2001, págs. 28-29)

Estos aprendizajes virtuales estan configurados con herramientas que permitan acercar y entrelazar a los protagonistas de la investigación, información o comunicación. Desde esta condición las redes sociales ofrecen múltiples alternativas para su uso con fines pedagógicos, como son los grupos de inter-aprendizaje, compartir información, trabajo colaborativo, crear espacios de discusión, espacios para la metacognición, entre otros (Barba Téllez \& Palacios Trujillo, 2018).

Por lo tanto, el objetivo general de esta investigación es analizar el uso de las redes sociales para actividades académicas colaborativas en la educación superior. La metodología empleada para el desarrollo fue investigación documental y bibliográfica.

\section{Método.}

Las redes sociales son herramientas que permiten generar mayor conocimiento y en mayor cantidad poblacional que los métodos pedagógicos tradicionales por lo que su importancia radica en la formación de individuos profesionales en su área, pero también con amplio carácter social. Por lo cual, la metodología empleada para obtener estas características es la de investigaciones de tipo documental y bibliográfica a través de la utilización de medios como textos, documentos y artículos científicos publicados disponibles en la web. 


\section{Uso de las redes sociales para actividades académicas colaborativas en la educación superior}

Vol. 3, núm. 3., (2019) Mónica Patricia Acurio Acurio; José Danilo Villares Pazmiño; Paulino Javier Suarez Guamán

\section{Resultados.}

Características de las TIC

Uno de los principales avances del desarrollo de la humanidad es la tecnología en todas las áreas de las ciencias. Esto ha permitido que los procesos sean realizados en corto tiempo y con una mayor precisión y exactitud. La educación no escapa de esta tecnología cambiante lo cual ha permitido crear nuevas formas de endeñar y aprender a través del uso de las diversas herramientas que brinda. La virtualidad, más que la simple incorporación de las tecnologías digitales, implica la adopción de nuevos métodos pedagógicos, nuevas actitudes y hábitos ante el aprendizaje (Facundo, 2004).

Estas tecnologías digitales van de la mano con la implementación de las TIC, las cuales han aparecido por los avances en las telecomunicaciones. Las TIC son el conjunto de tecnologías que permiten el acceso, producción, tratamiento y comunicación de información presentada en diferentes códigos (texto, imagen, sonido) (Ortí, 2011). Por lo que, es necesario poder determinar la funcionabilidad de esta herramienta en el ámbito educativo tal cual se puede observar en la Tabla 1. 


\section{Uso de las redes sociales para actividades académicas colaborativas en la educación superior}

Vol. 3, núm. 3., (2019)

Mónica Patricia Acurio Acurio; José Danilo Villares Pazmiño; Paulino Javier Suarez Guamán

Tabla 1. Funciones de las TIC en la educación

- Medio de expresión: escribir, dibujar, presentaciones, webs.

- Canal de comunicación, colaboración e intercambio.

- Instrumento para procesar la información.

- Fuente ablerta de información (mass media, self media)

- Instrumento para la gestión administrativa y tutorial.

- Herramienta de diagnóstico y rehabilitación.

- Medio didáctico: informa, entrena, guia aprendizaje, motiva

- Generador de nuevos escenarios formativos

- Medio lúdico y para el desarrollo cognitivo.

- Contenido curricular: conocimientos, competencias

Fuente: (Graells, 2013)

Al poder analizar estas funciones de las TIC dentro de la educación entonces es necesario también analizar cómo es la relación con las estrategias de enseñanza-aprendizaje implementadas por los docentes. Esta relación debe ser vinculativa ya que deben ser vistos como medios para ayudar a fomentar los métodos didácticos en el aula de clase. Las TIC no son más que medios y recursos que se pueden utilizar en el proceso didáctico, por lo que como se utilice, para qué y en qué contexto es lo que hace que tengan una incidencia u otra (Rodríguez-Izquierdo, 2011).

Ahora poder cumplir con estas expectativas de enseñanza y aprendizaje depende de varios factores que puedan cumplir con estos objetivos. En primer lugar, los usos que los participantes hagan efectivamente de las TIC dependerán en buena medida de la naturaleza y características del equipamiento y de los recursos tecnológicos puestos a su disposición (Coll, 


\section{Uso de las redes sociales para actividades académicas colaborativas en la educación superior}

Vol. 3, núm. 3., (2019)

Mónica Patricia Acurio Acurio; José Danilo Villares Pazmiño; Paulino Javier Suarez Guamán 2009). Esto va de la mano con políticas en el sector gubernamental y educativo que permita poder cumplir con las expectativas.

"Para que pueda haber un verdadero impacto de las TIC en la configuración de nuevos modos de enseñanza y aprendizaje se requiere de una visión integradora de las políticas educativas, la organización de la institución, recursos materiales y actores involucrados que se inscriban en el desarrollo de un proyecto educativo claramente definido y compartido". (Castro, Guzmán, \& Casado, 2007, pág. 230)

En el Ecuador se han implementado estrategias que puedan vincular estas TIC con el sistema educativo ya que se ha comprendido que el internet es una herramienta que permite que las sociedades estén conectadas y puedan obtener una información rápida y veraz.

"Por esto es indispensable que el gobierno ecuatoriano pueda cumplir con el objetivo de la Agenda Educativa Digital que es lograr que todas las escuelas del país cuenten con internet, pero aunque es vital la implementación de esta nueva cultura digital, también es importante que todos los docentes ecuatorianos tengan como competencia el conocimiento y el manejo de las herramientas tecnológicas dentro de los salones de clases". (Navarrete Mendieta \& Mendieta García, 2018, págs. 133-134)

Estos planes van enmarcados en el desarrollo de la educación digital a través de los centros de educación primaria y secundaria. La educación universitaria no puede estar ausente ante las nuevas TIC, permaneciendo desconectada del mundo social, ajena a los cambios, desvinculada de su entorno inmediato, obsoleta en recursos humanos y materiales ya que, de lo contrario, estaría condenada al fracaso (Briceño, 2014). 


\section{Uso de las redes sociales para actividades académicas colaborativas en la educación superior}

Vol. 3, núm. 3., (2019)

Mónica Patricia Acurio Acurio; José Danilo Villares Pazmiño; Paulino Javier Suarez Guamán

Poder enfrentar estas realidades es de vital importancia, pero para ello se necesita el compromiso de todos los factores que puedan incidir en que estas tecnologías sean aplicables y efectivas dentro de la sociedad. Es aquí donde puede visualizar 4 escenarios donde las instituciones de educación superior se pueden desenvolver. Dichos posibles escenarios se configuran en torno a dos ejes principales sobre los que pueden tomarse unas u otras decisiones: la centralización o descentralización de los servicios y el peso de la responsabilidad en términos de calidad educativa (Quintero, 2009). Esto se puede visualizar en la Figura 2. 


\section{Uso de las redes sociales para actividades académicas colaborativas en la educación superior}

Vol. 3, núm. 3., (2019)

Mónica Patricia Acurio Acurio; José Danilo Villares Pazmiño; Paulino Javier Suarez Guamán

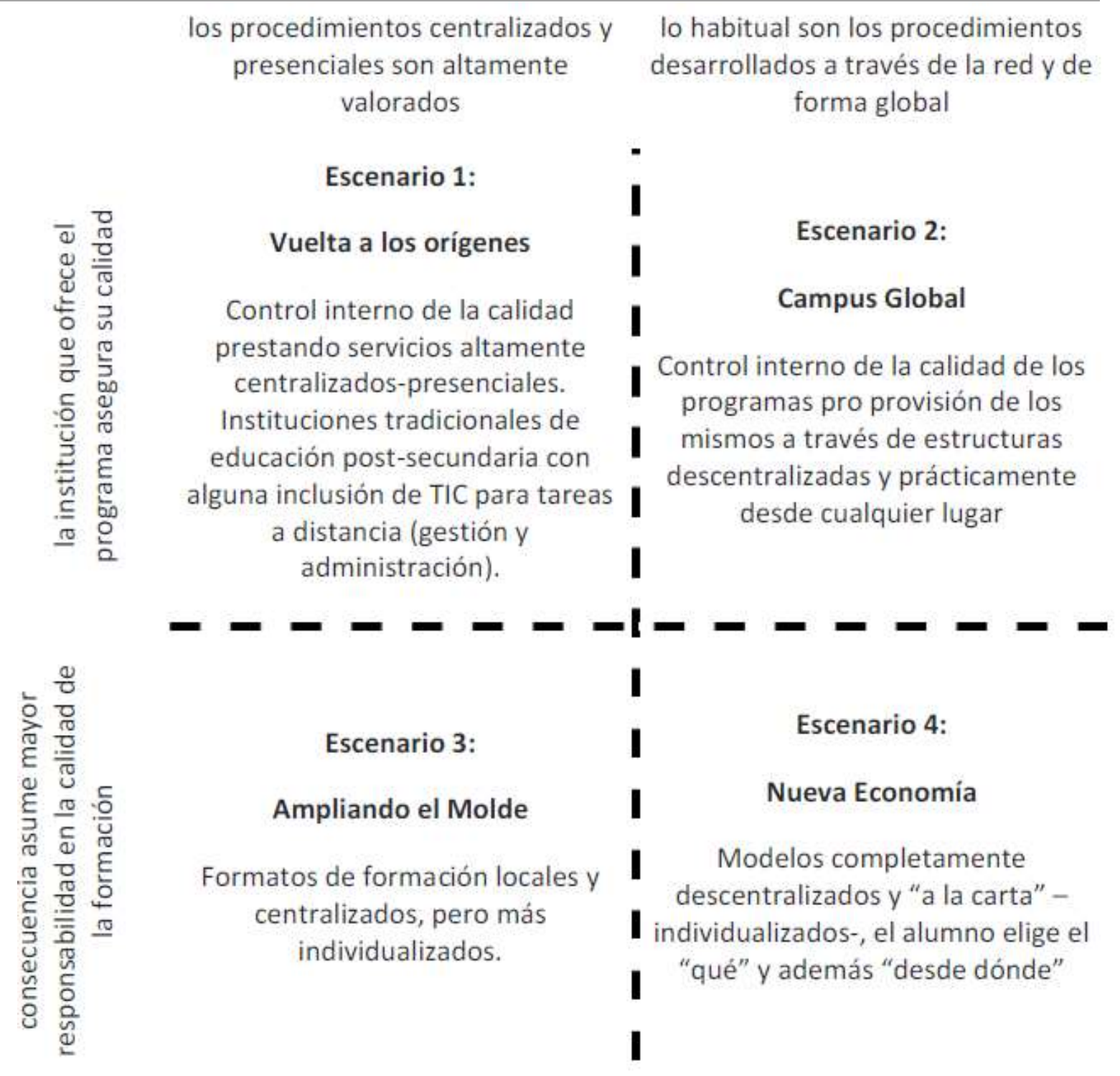

Figura 2. Cuatro escenarios para el desarrollo educativo

Fuente: (Quintero, 2009)

La incorporación de las TIC en el ámbito educativo debe ir de la mano con 3 aspectos a los cuales depende de la potencialidad educativa que se le atribuye a estas herramientas. Estos aspectos son los siguientes: 


\section{Uso de las redes sociales para actividades académicas colaborativas en la educación superior}

Vol. 3, núm. 3., (2019)

Mónica Patricia Acurio Acurio; José Danilo Villares Pazmiño; Paulino Javier Suarez Guamán

"Las TIC se contemplan como herramientas de comunicación y de búsqueda, acceso, procesamiento y difusión de la información cuyo conocimiento y dominio es absolutamente necesario en la sociedad actual; tratar de incorporar las TIC a la educación escolar con el fin de hacer más eficientes y productivos los procesos de enseñanza y aprendizaje, aprovechando los recursos y posibilidades que ofrecen estas tecnologías; y considerar las TIC como instrumentos mediadores de los procesos intra e interpsicológicos implicados en la enseñanza y en el aprendizaje”. (Coll C. , 2008, págs. 17-19)

Realidades de las universidades ecuatorianas con las TIC

Para poder vincular estas herramientas tecnológicas dentro de las universidades es necesario poder aplicar una serie de reglamentos, estatutos y leyes que permitan que pueda ser sostenible en el tiempo ya que no basta con el solo compromiso de los actores que desean fomentar el conocimiento y la investigación. La calidad de este vínculo va depender de características que se lo permitan:

“Ser operacional, es decir poder traducirse en elementos fácilmente manejables dentro de una guía, modelo o procedimiento de evaluación; Que abarque de una u otra forma las funciones sustantivas de la universidad; Que lleve implícito el concepto de apreciación o evaluación; y que esté ligado a la pertinencia social”. (Cabrera, 2005, pág. 5)

Para que se pueda cumplir con estos objetivos es preciso entonces poder definir el carácter general de la educación superior como un bien público. Esto quiere decir que no tiene fines de lucro, no es mercantilizable, debe perseguir el interés general y reviste un impacto que 


\section{Uso de las redes sociales para actividades académicas colaborativas en la educación superior}

Vol. 3, núm. 3., (2019)

Mónica Patricia Acurio Acurio; José Danilo Villares Pazmiño; Paulino Javier Suarez Guamán rebasa el beneficio individual, por lo que permite la inserción soberana del Ecuador en el contexto de las naciones (Ramírez \& Minteguiada, 2010).

Esto siembra las bases de un cambio en la dirección que debe tomar la educación superior universitaria que no solo involucra políticas de estado sino también compromiso de quienes quieren protagonizar el cambio. La construcción de un sistema de innovación y las transformaciones en el campo de la educación superior puede ser un objetivo loable también las transformaciones en el sistema cognitivo se vuelven necesarias para construir el tipo de sociedad que se desea (Gallegos, 2013).

“En el Ecuador existe políticas que disponen la inclusión en la educación del uso de la tecnología, sin embargo, el incorporar la educación con la tecnología aún es un efecto no instaurado, así pues, es importante el considerar la regulación estatal y la finalidad que se pretende obtener el facilitar el proceso de enseñanza-aprendizaje que genere motivación, participación, y evaluación de los resultados académicos con la inclusión de recursos tecnológicos”. (Peralta, Martinez, \& Sulca, 2017, pág. 421)

Poder generar un proceso de enseñanza y aprendizaje, dentro del uso de las TIC, depende de parámetros como las políticas gubernamentales y del compromiso de los protagonistas de la generación de investigación y conocimiento. Es por ello, que ahí la necesidad de recursos humanos y herramientas de gestión en ciencia, tecnología e innovación, que permitan mejorar la evaluación de los programas e instrumentos promoviendo una adecuación inteligente de los mismos a los objetivos planteados (Milia, 2014). 


\section{Uso de las redes sociales para actividades académicas colaborativas en la educación superior}

Vol. 3, núm. 3., (2019)

Mónica Patricia Acurio Acurio; José Danilo Villares Pazmiño; Paulino Javier Suarez Guamán

Es decir, que el parámetro fundamental para el desarrollo de estas tecnologías pedagógicas es el carácter humano y el compromiso de ellas. Por lo cual, urge la necesidad del uso de herramientas tecnológicas en el proceso de enseñanza-aprendizaje, con el fin de tener docentes motivados y comprometidos con su profesión, y alumnos que adquieran nuevo conocimiento a través del uso de la tecnología (López \& Constante, 2018). Para ello se necesita que los involucrados tengan accesos a equipos tecnológicos como PC, tablets, teléfonos inteligentes, entre otros como se muestra en la Tabla 2. Ahora estos equipos requieren del acceso a internet con el fin de que sirva de puente entre los docentes y estudiantes que desean desarrollar el conocimiento.

Tabla 2. Equipamiento tecnológico del hogar en el Ecuador

\begin{tabular}{cccccc}
\hline Periodo & $\begin{array}{c}\text { Computadora de } \\
\text { escritorio }\end{array}$ & $\begin{array}{c}\text { Computadora } \\
\text { portátil }\end{array}$ & $\begin{array}{c}\text { Computadora de } \\
\text { escritorio y } \\
\text { portátil }\end{array}$ & Telefonía fija & $\begin{array}{c}\text { Telefonía } \\
\text { celular }\end{array}$ \\
\hline dic-07 & $20,3 \%$ & - & - & $35,5 \%$ & - \\
dic-08 & $22,8 \%$ & - & - & $37,1 \%$ & - \\
dic-09 & $23,4 \%$ & - & - & $35,6 \%$ & - \\
dic-10 & $24,0 \%$ & $9,0 \%$ & $6,0 \%$ & $38,5 \%$ & $49,7 \%$ \\
dic-11 & $24,7 \%$ & $9,8 \%$ & $5,8 \%$ & $39,9 \%$ & $78,8 \%$ \\
dic-12 & $26,4 \%$ & $13,9 \%$ & $8,1 \%$ & $42,4 \%$ & $81,7 \%$ \\
dic-13 & $27,5 \%$ & $18,1 \%$ & $9,9 \%$ & $39,6 \%$ & $86,4 \%$ \\
dic-14 & $27,1 \%$ & $20,6 \%$ & $10,2 \%$ & $38,2 \%$ & $89,7 \%$ \\
dic-15 & $27,7 \%$ & $24,8 \%$ & $11,6 \%$ & $38,9 \%$ & $89,5 \%$ \\
dic-16 & $26,7 \%$ & $27,6 \%$ & $12,0 \%$ & $38,4 \%$ & $90,1 \%$ \\
dic-17 & $25,9 \%$ & $26,0 \%$ & $11,2 \%$ & $36,9 \%$ & $90,7 \%$ \\
\hline
\end{tabular}

Fuente: (ENEMDU, 2018)

Nota: (-) Información no disponible. Computadora portátil incluye laptop y Tablet.

Tabla 3. Porcentaje de hogares que tienen acceso a internet en el Ecuador. 


\section{Uso de las redes sociales para actividades académicas colaborativas en la educación superior}

Vol. 3, núm. 3., (2019)

Mónica Patricia Acurio Acurio; José Danilo Villares Pazmiño; Paulino Javier Suarez Guamán

\begin{tabular}{cccc}
\hline Periodo & Nacional & Urbana & Rural \\
\hline dic-08 & $7,0 \%$ & $9,9 \%$ & $0,9 \%$ \\
dic-09 & $7,7 \%$ & $11,0 \%$ & $0,7 \%$ \\
dic-10 & $11,8 \%$ & $16,7 \%$ & $1,3 \%$ \\
dic-11 & $16,9 \%$ & $23,7 \%$ & $3,5 \%$ \\
dic-12 & $22,5 \%$ & $31,4 \%$ & $4,8 \%$ \\
dic-13 & $28,3 \%$ & $37,0 \%$ & $9,1 \%$ \\
dic-14 & $32,4 \%$ & $40,4 \%$ & $14,6 \%$ \\
dic-15 & $32,8 \%$ & $41,0 \%$ & $13,7 \%$ \\
dic-16 & $36,0 \%$ & $44,6 \%$ & $16,4 \%$ \\
dic-17 & $37,2 \%$ & $46,1 \%$ & $16,6 \%$ \\
\hline
\end{tabular}

Fuente: (ENEMDU, 2018)

"Y aunque la Unesco sostiene que los teléfonos móviles y tablets pueden ser utilizados por estudiantes y docentes de todo el mundo, para acceder a información, simplificar la administración de datos y facilitar el aprendizaje de una forma nueva e innovadora, es indispensable que los docentes estén capacitador y formados en la metodología de la utilización de las TIC dentro de los salones para procurar el correcto uso de estos dispositivos". (Navarrete Mendieta \& Mendieta García, 2018, pág. 134)

Ya con estas herramientas y con el recurso humano disponible entonces es necesario que la sociedad quiebre los viejos paradigmas incrustados sobre la forma pedagógica de la educación. Esto quiere decir que mientras más temprano se familiarice el ciudadano con ellas mayor el nivel de conocimiento que pueden aportar, sin olvidar que el uso de tecnologías implica inversión de alguna parte y eso está reflejado en la Tabla 1 (Mestanza, Caguana, \& Espinoza, 2018).

Ya las políticas universitarias empezaron a desarrollar sus plataformas funcionales a través del uso de estas herramientas digitales a través de la implementación de paginas web de las universidades y de los correos electrónicos institucionales. Esto se consolida al establecer 


\section{Uso de las redes sociales para actividades académicas colaborativas en la educación superior}

Vol. 3, núm. 3., (2019)

Mónica Patricia Acurio Acurio; José Danilo Villares Pazmiño; Paulino Javier Suarez Guamán

procesos y herramientas que soportan al Sistema de Difusión junto al compromiso, uso y

conocimiento de los gestores para garantizar una difusión efectiva (Cueva, 2016).

\section{Las redes sociales y las universidades ecuatorianas}

La enseñanza-aprendizaje debe trascender de los viejos paradigmas educativos y masificarlos para que la sociedad sea protagonista de la investigación y conocimiento que se genere. Debe vencer las barreras estigmatizadas y abrir las puertas a un mundo globalizado. Es trascender en los viejos esquemas evaluativos y empezar a crear nuevas formas de generar un contenido. Sin embargo, las reformas en el ámbito educativo superior ofrecen con frecuencia resistencia, incertidumbre, inseguridad, lo que está basado fundamentalmente en la tradición de años y en posturas universitarias rígidas y rutinarias (Salinas, Alcívar, Gómez Cabrera, \& Aguilar Salazar, 2016).

Pero, la educación superior ha empezado a tomar en consideración esas herramientas tecnológicas para ser aplicadas en la generación del conocimiento. La funcionabilidad no ha sido la correcta al principio. Se consideraba que la educación virtual era la utilización de esos medios para presentar los viejos esquemas didácticos y no como las herramientas que sirvan de apoyo a esa nueva generación de conocimientos.

"No se utiliza todo el potencial que brindan las tecnologías digitales para buscar información, rompiendo las restricciones de distancia, tiempo, y hasta de la propia realidad, para aprender investigando, para visualizar desde las más diversas dimensiones, para utilizar la comunicación y la colaboración como forma de enriquecer los procesos de aprendizaje o para que el estudiante asuma la responsabilidad de un proceso de aprendizaje autónomo, 


\section{Uso de las redes sociales para actividades académicas colaborativas en la educación superior}

Vol. 3, núm. 3., (2019) Mónica Patricia Acurio Acurio; José Danilo Villares Pazmiño; Paulino Javier Suarez Guamán consciente, permanente y dirigido, en lo fundamental, por él mismo". (Facundo, 2004, pág.

Poder conseguir este objetivo del aprendizaje autónomo, consciente, permanente y dirigido por parte del individuo que desea afrontar esta realidad se requiere de ciertas herramientas físicas que se lo permitan como lo es el equipamiento tecnológico (ver Tabla 2), tener acceso a internet (ver Tabla 3) y que ese internet pueda ser usado en cualquier lugar donde se encuentre como se puede observar en la Tabla 4.

Tabla 4. Porcentaje de lugares de uso del internet a nivel nacional en el Ecuador

\begin{tabular}{lcccccc}
\hline Periodo & Hogar & Trabajo & $\begin{array}{c}\text { Institución } \\
\text { Educativa }\end{array}$ & $\begin{array}{c}\text { Centros de } \\
\text { acceso } \\
\text { público }\end{array}$ & $\begin{array}{c}\text { Casa de } \\
\text { otra } \\
\text { persona }\end{array}$ & Otro \\
\hline dic-08 & $17,0 \%$ & $15,7 \%$ & $14,5 \%$ & $51,5 \%$ & $1,1 \%$ & $0,3 \%$ \\
dic-09 & $24,9 \%$ & $14,6 \%$ & $12,9 \%$ & $45,9 \%$ & $1,3 \%$ & $0,4 \%$ \\
dic-10 & $31,1 \%$ & $13,4 \%$ & $13,4 \%$ & $40,1 \%$ & $1,3 \%$ & $0,6 \%$ \\
dic-11 & $34,0 \%$ & $11,9 \%$ & $13,7 \%$ & $38,3 \%$ & $1,6 \%$ & $0,6 \%$ \\
dic-12 & $41,8 \%$ & $10,7 \%$ & $11,0 \%$ & $34,2 \%$ & $1,6 \%$ & $0,8 \%$ \\
dic-13 & $45,1 \%$ & $9,8 \%$ & $12,2 \%$ & $29,8 \%$ & $2,1 \%$ & $1,0 \%$ \\
dic-14 & $46,7 \%$ & $8,6 \%$ & $8,8 \%$ & $28,2 \%$ & $3,2 \%$ & $4,5 \%$ \\
dic-15 & $51,7 \%$ & $8,6 \%$ & $7,5 \%$ & $27,5 \%$ & $3,6 \%$ & $1,0 \%$ \\
dic-16 & $54,1 \%$ & $8,9 \%$ & $7,7 \%$ & $23,2 \%$ & $4,4 \%$ & $1,6 \%$ \\
dic-17 & $57,2 \%$ & $9,2 \%$ & $7,3 \%$ & $19,9 \%$ & $5,2 \%$ & $1,2 \%$ \\
\hline
\end{tabular}

Fuente: (ENEMDU, 2018)

Ahora, la sociedad ecuatoriana tiene acceso a internet para obtener algún tipo de información, es decir, busca investigar contenidos que le sean de interés. Esto quiere decir que la sociedad tiene una razón y uso de la internet basada en la búsqueda de alguna información, para poder comunicarse, por educación y aprendizaje, entre otros. Esto se puede reflejar en la Tabla 5. 


\section{Uso de las redes sociales para actividades académicas colaborativas en la educación superior}

Vol. 3, núm. 3., (2019)

Mónica Patricia Acurio Acurio; José Danilo Villares Pazmiño; Paulino Javier Suarez Guamán

Indudablemente que la población ecuatoriana ha tenido un crecimiento en el contacto y manejo de estas herramientas electrónicas en una gran variedad de formas y necesidades.

Tabla 5. Razones del uso del internet de las personas a nivel nacional

\begin{tabular}{cccccc}
\hline Periodo & $\begin{array}{c}\text { Obtener } \\
\text { información }\end{array}$ & $\begin{array}{c}\text { Comunicación } \\
\text { en general }\end{array}$ & $\begin{array}{c}\text { Educación y } \\
\text { aprendizaje }\end{array}$ & $\begin{array}{c}\text { Razones de } \\
\text { trabajo }\end{array}$ & Otro \\
\hline dic-08 & $23,4 \%$ & $23,9 \%$ & $40,1 \%$ & $7,3 \%$ & $5,3 \%$ \\
dic-09 & $30,0 \%$ & $18,9 \%$ & $38,7 \%$ & $7,8 \%$ & $4,6 \%$ \\
dic-10 & $27,2 \%$ & $22,4 \%$ & $40,0 \%$ & $5,3 \%$ & $5,0 \%$ \\
dic-11 & $31,1 \%$ & $32,6 \%$ & $29,1 \%$ & $3,4 \%$ & $3,8 \%$ \\
dic-12 & $36,0 \%$ & $28,2 \%$ & $27,5 \%$ & $3,8 \%$ & $4,4 \%$ \\
dic-13 & $32,0 \%$ & $25,5 \%$ & $31,7 \%$ & $4,9 \%$ & $6,0 \%$ \\
dic-14 & $35,2 \%$ & $30,9 \%$ & $26,5 \%$ & $2,9 \%$ & $4,5 \%$ \\
dic-15 & $36,9 \%$ & $29,4 \%$ & $25,4 \%$ & $4,1 \%$ & $4,2 \%$ \\
dic-16 & $38,0 \%$ & $31,5 \%$ & $23,2 \%$ & $3,6 \%$ & $3,7 \%$ \\
dic-17 & $40,7 \%$ & $31,0 \%$ & $21,1 \%$ & $3,3 \%$ & $3,9 \%$ \\
\hline
\end{tabular}

Fuente: (ENEMDU, 2018)

"Resulta pertinente, el poder conocer las tendencias de uso y el acceso; tanto a dispositivos electrónicos (ordenadores, tabletas y teléfonos inteligentes), como a las herramientas web por parte de los estudiantes universitarios, lo cual permitirá contar con las plataformas y los recursos tecnológicos que sean factibles de ser adoptados por los estudiantes; de cara a la implementación de sus entornos personales de aprendizaje móviles". (Humanante Ramos, García-Peñalvo, Conde González, \& Velasco-Silva, 2015, págs. 287288)

Estos entornos de aprendizajes móviles se pueden ofrecer a través de aulas virtuales y recursos online como las redes sociales. Para ello la población ecuatoriana debe tener un acceso a 


\section{Uso de las redes sociales para actividades académicas colaborativas en la educación superior}

Vol. 3, núm. 3., (2019)

Mónica Patricia Acurio Acurio; José Danilo Villares Pazmiño; Paulino Javier Suarez Guamán las redes sociales y por ende la utilización de equipos de telefonía celular inteligente que le permita poder obtener la información en cualquier momento y espacio. Esto se puede visualizar en la Tabla 6.

Tabla 6. Población con celular inteligente y redes sociales en el Ecuador

\begin{tabular}{ccccc}
\hline Período & $\begin{array}{c}\text { Población } \\
\text { Total }\end{array}$ & $\begin{array}{c}\text { Población que } \\
\text { tiene teléfono } \\
\text { celular activado }\end{array}$ & $\begin{array}{c}\text { Población que } \\
\text { tiene teléfono } \\
\text { celular } \\
\text { SMARTPHONE }\end{array}$ & $\begin{array}{c}\text { Población que en } \\
\text { su teléfono } \\
\text { smartphone } \\
\text { utiliza redes } \\
\text { sociales }\end{array}$ \\
\hline dic-11 & 14.478 .129 & 6.209 .858 & 522.640 & 365.427 \\
dic-12 & 14.682 .556 & 6.859 .938 & 839.705 & 641.914 \\
dic-13 & 15.872 .755 & 7.453 .781 & 1.261 .944 & 1.081 .620 \\
dic-14 & 16.148 .648 & 7.820 .597 & 1.928 .108 & 1.722 .159 \\
dic-15 & 16.404 .531 & 8.174 .520 & 3.084 .886 & 2.807 .282 \\
dic-16 & 16.714 .929 & 8.482 .236 & 4.484 .087 & 4.224 .984 \\
dic-17 & 16.961 .926 & 8.987 .750 & 5.716 .770 & 5.412 .575 \\
\hline
\end{tabular}

Fuente: (ENEMDU, 2018)

Es por ello que es necesario poder definir el concepto de redes sociales para poder comprender su importancia en la masificación del conocimiento que se pueda generar dentro de las universidades.

"Una red social es una estructura social que se puede representar en forma de uno o varios grafos en el cual los nodos representan individuos (a veces denominados actores) y las aristas relaciones entre ellos. Las relaciones pueden ser de distinto tipo, como intercambios financieros, amistad, relaciones sexuales, o rutas aéreas. También es el medio de interacción de distintas personas como juegos en línea, chats, foros”. (Tamayo, 2012, pág. 136) 


\section{Uso de las redes sociales para actividades académicas colaborativas en la educación superior}

Vol. 3, núm. 3., (2019)

Mónica Patricia Acurio Acurio; José Danilo Villares Pazmiño; Paulino Javier Suarez Guamán

Las redes sociales dentro de las universidades, como mecanismo de formación y generación de conocimiento, juega un papel importante ya que se considera amigable para los protagonistas de los hechos y también de que su uso es mucho más digerible que otros tipos de herramientas tecnológicas. Por lo que, las redes sociales en las universidades ecuatorianas se constituyen en herramientas de generación y retroalimentación de información, en donde el modelo de comunicación resulta eficiente e innovador para el contexto universitario (Téllez \& Trujillo, 2018).

Sin duda alguna, las redes sociales están jugando un papel importante en la formación de la nueva sociedad ya que acerca al individuo a innumerables informaciones que pueden ayudar a la formación del conocimiento. Las redes sociales tienen el innegable valor de acercar el aprendizaje informal y el formal, ya que permiten al alumno expresarse por sí mismo, entablar relaciones con otros, así como atender a las exigencias propias de su educación (Tamayo, 2012).

"Las redes sociales propician un proceso de construcción sociocultural en el que cada miembro aprende más de lo que aprendería por sí mismo, debido a la interactividad con otros miembros de su grupo. Desde esta perspectiva, el resultado de un trabajo en redes sociales colaborativas puede tener un valor agregado muy superior al que pudiera tener la sumatoria de los trabajos individuales realizados de manera independiente por cada miembro dentro de un grupo presencial, por lo que se consideran una poderosa herramienta capaz de transformar los procesos de aprendizajes, todo depende de su empleo y finalidades". (Téllez \& Trujillo, 2018, págs. 86-87) 


\section{Uso de las redes sociales para actividades académicas colaborativas en la educación superior}

Vol. 3, núm. 3., (2019)

Mónica Patricia Acurio Acurio; José Danilo Villares Pazmiño; Paulino Javier Suarez Guamán Dentro de las redes sociales se encuentra el WhatsApp, Facebook, Twitter y Youtube como las herramientas más utilizadas dentro de las universidades ecuatorianas. Facebook y Twitter poseen alto grado de aceptación como medio de comunicación institucional, y por eso integran al sistema de difusión, considerando la naturaleza de la información como pública: sin embargo, el sistema puede reemplazarlas por otras TIC, según las necesidades y tendencias (Cueva, 2016). La red social más utilizada, WhatsApp, que puede considerarse más como un servicio de mensajería, evidencia un escaso aprovechamiento de las redes sociales como recursos para recuperar contenidos en la Red y generar conocimiento a partir de un aprendizaje cooperativo y colaborativo (Martínez-Sala \& Alemany-Martínez, 2018).

También se ha comprobado que el poco uso o la nulidad del mismo han tenido efectos negativos en el desarrollo del conocimiento y es especial en la formación del profesional ecuatoriano. El menor uso de redes sociales en el ámbito académico revela carencias en los alumnos y alumnas al respecto de su capacitación para evaluar su comportamiento informacional en ambientes colaborativos (comunidades en línea) y en medios sociales (Martínez-Sala \& Alemany-Martínez, 2018). Se ha comprobado que contribuyen a fomentar el autoaprendizaje de conocimientos, habilidades y valores para la vida, el pensamiento crítico, la aceptación a la diversidad, entre otras cuestiones necesarias al nuevo ciudadano que necesita la sociedad ecuatoriana (Téllez \& Trujillo, 2018).

\section{Discusión y conclusiones.}

Una de las características fundamentales para poder desarrollar un nuevo ciudadano es poder aplicar una serie de herramientas en las cuales se fomente el conocimiento de manera que 


\section{Uso de las redes sociales para actividades académicas colaborativas en la educación superior}

Vol. 3, núm. 3., (2019)

Mónica Patricia Acurio Acurio; José Danilo Villares Pazmiño; Paulino Javier Suarez Guamán

el individuo tenga la oportunidad de poder escoger cual le es más beneficiosos para su caso en

específico. Es decir, debe aplicar una investigación que le permita discernir de las alternativas de las cuales está siendo partidario. Es por ello que la investigación ha formado parte fundamental en el desarrollo de la humanidad. Desde pasa de una edad de piedra a la edad de bronce está involucrada la investigación como proceso para satisfacer las necesidades mínimas de la sociedad. La investigación ha sido parte del proceso evolutivo de la humanidad y por ende el sistema educativo no debe escapar a ella. Existen diversos mecanismos pedagógicos que han ayudado a fomentar el crecimiento de la investigación. Partiendo de la evolución de los enfoques educativos, pasando de conductivista a constructivista, la investigación ha formado parte de este desarrollo.

Para poder realizar las investigaciones y con ello poder cosechar los conocimientos es necesario ir avanzando en las herramientas pedagógicas. Este paso a sido difícil por los paradigmas incrustados en la sociedad educativa. El formalismo pedagógico ha tenido un papel importante en el desarrollo del conocimiento y permitir que otras herramientas y métodos puedan ocupar su espacio ha sido una tarea difícil. En los últimos tiempos, han aparecido las tecnologías de la información y comunicación (TIC) como herramientas que ayudan a acercar más el conocimiento a través de investigaciones más digeribles y sencillas. Estas herramientas han vencido, a través del tiempo, las barreras paradigmáticas implementadas por la sociedad ya que esta ha tenido que verse involucrada debido al mundo globalizado cada vez más cambiante.

Es aquí donde estas TIC han jugado un papel importante en la masificación de la información y no es más que la utilización de las redes sociales como vehículo propulsor de esta 


\section{Uso de las redes sociales para actividades académicas colaborativas en la educación superior}

Vol. 3, núm. 3., (2019)

Mónica Patricia Acurio Acurio; José Danilo Villares Pazmiño; Paulino Javier Suarez Guamán generación de nuevos conocimientos. Las redes sociales han formado parte fundamental en el desarrollo educativo porque han permito un acercamiento de los protagonistas de la investigación y conocimiento ya que son herramientas más fáciles de controlar y manejar y de que pueden llegar a más lugares y expandirse a mayor población. Es decir, la cercanía que permiten estas herramientas ha hecho que la información llegue más rápida y en mayor cantidad que con los procesos tradicionales. Al igual, ha permitido formar un individuo profesional sino también un individuo social con amplio conocimiento de diversos ámbitos.

\section{Referencias.}

Area Moreira, M. (2010). ¿Por qué formar en competencias informacionales y digitales en la educación superior? RUSC. Universities and Knowledge Society Journal, vol. 7, núm. 2, $2-5$.

BAELO ÁLVAREZ, R., \& CANTÓN MAYO, I. (2009). Las tecnologías de la información y la comunicación en la educación superior. Estudio descriptivo y de revisión. Revista Iberoamericana de Educación. n. ${ }^{\circ}$ 50/7, 1-12.

Barba Téllez, M. N., \& Palacios Trujillo, E. P. (2018). LAS REDES SOCIALES EN EL CONTEXTO ACADÉMICO UNIVERSITARIO. DESAFIOS AL DOCENTE. Revista científica electrónica de Educación y Comunicación en la Sociedad del Conocimiento. Época II Año XVIII Número 18 Vol. I, 83-101.

Briceño, J. (2014). Reflexiones sobre la educación superior en Venezuela. Comunidad y Salud, $12(1), 69-73$.

Cabrera, V. A. (2005). El concepto calidad en la educación universitaria: clave para el logro de la competitividad institucional. Revista iberoamericana de Educación, 36(12), 1-7.

Carrizo Estévez, J. D. (2010). Importancia de la investigación en la formación de Pregrado. Panorama Cuba y Salud, vol. 5, núm. 3, 3-4.

Castro, S., Guzmán, B., \& Casado, D. (2007). Las Tic en los procesos de enseñanza y aprendizaje. Laurus, 13(23), 213-234.

Clark, B. R. (1998). Crecimiento sustantivo y organización innovadora: nuevas categorías para la investigación en educación superi. Perfiles Educativos, núm. 81, 1-16. 


\section{Uso de las redes sociales para actividades académicas colaborativas en la educación superior}

Vol. 3, núm. 3., (2019)

Mónica Patricia Acurio Acurio; José Danilo Villares Pazmiño; Paulino Javier Suarez Guamán

Coll, C. (2008). Aprender y enseñar con las TIC: expectativas, realidad y potencialidades. Boletín de la Institución Libre de Enseñanza, 72, 17-40.

Coll, C. (2009). Aprender y enseñar con las TIC: expectativas, realidad y potencialidades. En R. Carneiro, J. C. Toscano, \& T. Díaz, Los desafíos de las TIC para el cambio educativo. (págs. 113-126). Madrid - España: Fundación Santillana.

Cueva, M. C. (2016). Las Tecnologías de la Información y la Comunicación como Medio de Difusión Universitaria. Hallazgos21, develando ciencia por el desarrollo, 1(2), 172-180.

ENEMDU. (2018). Tecnologías de la Información y Comunicación. Quito - Ecuador: Instituto Nacional de Estadística y Censos.

Facundo, Á. H. (2004). La virtualización desde la perspectiva de la modernización de la educación superior: consideraciones pedagógicas. International Journal of Educational Technology in Higher Education (ETHE), 1(1), 1-17.

Gallegos, R. (2013). Tercera ola de transformación de la educación superior en Ecuador. Quito - Ecuador: Secretaría Nacional de Educación Superior, Ciencia, Tecnología e Innovación.

GIL PEREZ, D. (1983). TRES PARADJGMAS BASICOS EN LA ENSENANZA DE LAS CIENCIAS. ENSENANZA DE LAS CIENCIAS, 26-33.

Graells, P. M. (2013). Impacto de las TIC en la educación: funciones y limitaciones. 3C TIC, $2(1), 1-15$.

Humanante Ramos, P. R., García-Peñalvo, F. J., Conde González, M. Á., \& Velasco-Silva, D. P. (2015). Diagnóstico del uso de los dispositivos electrónicos y de las herramientas web 2.0 desde un enfoque PLE en un grupo de estudiantes de ingeniería. Madrid - España: III Congreso Internacional sobre Aprendizaje, Innovación y Competitividad (CINAIC 2015).

López Noguero, F. (2002). El análisis de contenido como método de investigación. Revista de Educación - Universidad de Huelva, 13., 167-179.

López, Y. A., \& Constante, G. F. (2018). Las TIC como herramienta de apoyo didáctico en el proceso de enseñanza-aprendizaje. Revista Científica Cátedra, 1(1), 29-31.

Martínez Martín, M. (2006). FORMACIÓN PARA LA CIUDADANÍA Y EDUCACIÓN SUPERIOR. REVISTA IBEROAMERICANA DE EDUCACIÓN. N. ${ }^{\circ}$ 42, 85-102.

Martínez-Sala, A.-M., \& Alemany-Martínez, D. (2018). Integración eficiente de redes sociales como herramientas complementarias de aprendizaje y para la alfabetización digital en los estudios superiores de Publicidad y RR. PP. En R. Roig-Vila, El compromiso académico 


\section{Uso de las redes sociales para actividades académicas colaborativas en la educación superior}

Vol. 3, núm. 3., (2019)

Mónica Patricia Acurio Acurio; José Danilo Villares Pazmiño; Paulino Javier Suarez Guamán

y social a través de la investigación e innovación educativas en la Enseñanza Superior (págs. 1126-1136). Barcelona - España: Ediciones Octaedro.

Mestanza, R. C., Caguana, E. F., \& Espinoza, J. C. (2018). Plan Estratégico de Investigación, Desarrollo e Innovación para las TIC en el Ecuadori Propuesta viable para la inclusión? RECIAMUC, 2(1), 725-749.

Milia, M. F. (2014). Marco de Políticas Públicas de Ciencia, Tecnología y Educación Superior en el Ecuador. Nuevos horizontes: dinámicas y condicionamientos para una Investigación Universitaria de cara a la Sociedad. Buenos Aires, Argentina.: Congreso Iberoamericano de Ciencia, Tecnología, Innovación.

Navarrete Mendieta, G., \& Mendieta García, R. C. (2018). LAS TIC Y LA EDUCACIÓN ECUATORIANA EN TIEMPOS DE INTERNET: BREVE ANÁLISIS. Ales. Revista multidisciplinaria de investigación. Vol. 2 No. 15, 123-136.

Ortí, C. B. (2011). Las tecnologías de la información y comunicación (TIC). Univ. Val., Unidad Tecnol. Educ, (951), 1-7.

Ortiz Flores, E. P., \& Bernal Zepeda, M. (2007). Importancia de la incorporación temprana a la investigación científica en La Universidad de Guadalajara. Guadalajara - México.

Peralta, M. A., Martinez, J. V., \& Sulca, R. S. (2017). Fundamentos de aplicación de blendenlearning para las Universidades del Ecuador. Dominio de las Ciencias, 3(2), 401-423.

Pereira Pérez, Z. (2011). Los diseños de método mixto en la investigación en educación: Una experiencia concreta. Revista Electrónica Educare Vol. XV, $N^{\circ}$ 1, [15-29.

Quintero, L. J. (2009). Las universidades apostando por las TIC: modelos y paradojas de cambio institucional. EDUTEC. Revista Electrónica de Tecnología Educativa, (28), 105., 1-14.

Ramírez, R., \& Minteguiada, A. (2010). Transformaciones en la Educación Superior Ecuatoriana: Antecedentes y perspectivas futuras como consecuencias de la nueva constitución política. Revista Educación Superior y Sociedad (ESS) ISSN: 0798-1228, $15(1), 129-154$.

Ramos, C. A. (2015). LOS PARADIGMAS DE LA INVESTIGACIÓN CIENTÍFICA. Av.psicol. 23(1), 9-17.

Renés Arellano, P. (2018). PLANTEAMIENTO DE LOS ESTILOS DE ENSEÑANZA DESDE UN ENFOQUE COGNITIVO-CONSTRUCTIVISTA. TENDENCIAS PEDAGÓGICAS $N^{o} 31,47-67$. 


\section{Uso de las redes sociales para actividades académicas colaborativas en la educación superior}

Vol. 3, núm. 3., (2019)

Mónica Patricia Acurio Acurio; José Danilo Villares Pazmiño; Paulino Javier Suarez Guamán

Rodríguez-Izquierdo, R. M. (2011). Repensar la relación entre las TIC y la enseñanza universitaria: problemas y soluciones. Profesorado. Revista de curriculum y formación del profesorado. VOL. 15, $N^{o} 1,9-22$.

Rosenberg, M. (2001). E-learning. Strategies for Delivering Knowledge in the Digital Age. New York, NY: McGraw-Hill.

Salinas, Q., Alcívar, L., Gómez Cabrera, O. A., \& Aguilar Salazar, R. D. (2016). La innovación educativa en la Educación Superior ecuatoriana y el portafolio docente: instrumentos de desarrollo. Revista Cubana de Reumatología, 18(3), 297-303.

Tamayo, C. F. (2012). Plataformas virtuales como recurso para la enseñanza en la universidad: análisis, evaluación y propuesta de integración de moodle con herramientas de la web 2.0. Madrid - España: Doctoral dissertation, Universidad Complutense de Madrid.

Téllez, M. N., \& Trujillo, E. P. (2018). Las redes sociales en el contexto académico universitario. Desafios aldocente.Etic@ net, 18(1),83-101. 